\title{
Application of Lagrange Relaxation to Decentralized Optimization of Dispatching a Charging Station for Electric Vehicles
}

\author{
Shan Cheng ${ }^{1, *}$, Yichen Feng ${ }^{2}$ and Xianning Wang ${ }^{2}$ \\ 1 Hubei Provincial Collaborative Innovation Center for New Energy Microgrid, \\ China Three Gorges University, Yichang 443002, China \\ 2 College of Electrical Engineering and New Energy, China Three Gorges University, Yichang 443002, China; \\ yueshuimaoneko@126.com (Y.F.); 13997717090@163.com (X.W.) \\ * Correspondence: hpucquyzu@ctgu.edu.cn; Tel.: +86-136-1868-3042
}

Received: 10 December 2018; Accepted: 3 February 2019; Published: 4 March 2019

\begin{abstract}
To improve the computation efficiency of optimally dispatching large-scale cluster electric vehicles (EVs) and to enhance the profit of a charging station (CS) for EVs, this study investigates the optimal dispatch of the CS based on a decentralized optimization method and a time-of-use (TOU) price strategy. With the application of the Lagrange relaxation method (LRM), a decentralized optimization model with its solution is proposed that converts the traditional centralized optimization model into certain sub-problems. The optimization model aims to maximize the profit of CS, but it comprehensively considers the charging preference of EV users, the operation constraints of the distribution network, and the TOU strategy adopted by the CS. To validate the proposed decentralized optimal dispatching method, a series of numerical simulations were conducted to demonstrate its effect on the computation efficiency and stability, the profit of the CS, and the peak-load shifting. The result indicates that the TOU strategy markedly increases the profit of the CS in comparison with the fixed electricity price mechanism, and the computation efficiency and stability are much better than those of the centralized optimization method. Although it does not compensate the load fluctuation completely, the proposed method with the TOU strategy is helpful for filling the valley of power use.
\end{abstract}

Keywords: electric vehicles; decentralized optimization; Lagrange relaxation; TOU price

\section{Introduction}

Because of energy consumption and environmental protection issues, new energy vehicles [1] have attracted special attention from both industry and academics all over the world, and new energy vehicle technology is regarded as an important future transportation option. In China, to adjust and upgrade the industrial structure and realize sustainable development, a series of policies for new energy vehicles have been promulgated by the government in recent years. As one type of new energy vehicles, battery-based electric vehicles are promoted in large and medium-sized cities via a significant strategic policy of the Chinese government [2]. When acting as a charging load, the direct connection of battery-based electric vehicles to the power grid is not an effective approach for low-carbon development. In particular, uncontrolled charging of battery-based electric vehicles is more likely to result in a massive increase in the power load, which consequently generates a heavy burden on the electric power generation and transmission systems [3]. Such negative effects can be effectively relieved by charging the BEVs in charging stations (CSs) with centralized management. A CS is a collection of multiple chargers. If the CS is located in a micro-grid (MG) with renewable distributed resources, then charging battery-based electric vehicles in a CS is helpful for the utilization of these renewable distributed resources, which in turn promotes low-carbon development. Hence, to 
provide the charging service with good quality, realize the economic benefit of the CS, and reduce the negative effects on the security, stability, and economy of the distribution network operation resulted from the integration of the CS, one of the key problems is the scientific and reasonable dispatching of the CS.

To optimally dispatch the CS, a large number of optimization models and algorithms have been presented, most of which are classified into the centralized optimization. To optimally charge the batteries of the plug-in electric vehicles subject to usage constraints, Cortés and Martínez [4] proposed a hierarchical coordination algorithm that allows for adjustment of the charging strategy according to the price information and has a valley-filling profile for the power use. To minimize the total charging cost and reduce the power loss and voltage deviation of the distribution network, a novel centralized charging strategy for battery-based electric vehicles under the battery swapping scenario via a population-based heuristic approach was designed by Kang et al. [5]. However, the impact of different price schemes on the power load was not involved in [5]. Because the approach in [5] makes it inconvenient for some EVs to stop at a CS, Ou et al. [6] investigated the on-road wireless charging framework for EVs and studied their economic scheduling that predict the EV wireless charging demand and capture the dynamics of EV mobility. Instead of state-of-the-art charging scheduling based on open-loop strategies, an approximate dynamic programming feedback-based optimization method with continuous state space and action space was discussed in [7]. By considering both the transport and grid systems, Luo et al. [8] introduced a novel optimal charging scheduling strategy for different types of EVs that was proven to obtain improvements in both the transport system efficiency and the grid system operation.

Despite the effectiveness and advantages of the methods presented in [4-8], the commonality among them is the investigation of centralized optimization or control methods. With a small computation scale, the centralized method is able to rapidly identify the optimal solution. For optimizing the charging schedule of large-scale BEVs, the central controller is confronted with the cluster of communication and massive data processing burdens. It will result in long computation time, especially for the online optimal scheduling. To avoid such problems for scheduling the large-scale EVs charging optimally, researchers have investigated the decentralized and hierarchical method. In 2013, Karfopoulos and Hatziargyriou [9] proposed a multi-agent-based EV charging management system, and its efficiency was evaluated by numerical simulations of a realistic urban distribution network. Then, in 2016, Xydas et al. [10] presented another decentralized scheduling algorithm for adaptive EV charging that was also implemented via a multi-agent system and was proved to charge preferentially from renewable energy sources with the adaptive behavior of "responsive" EV agents. García-Triviño et al. [11] investigated a decentralized energy management system for a fast CS to control and operate the RESs. By considering the EV to be flexible mobile battery storage, Wang et al. [12] studied a dynamically updated electricity price-based decentralized scheduling strategy for the MG central controller. In [12], each MG is only required to solve its local problem and the total power exchange is limited within the safe range. To coordinate the queues among the highway CSs with only local information, Gusrialdi et al. [13] proposed a distributed algorithm to schedule EV flows and developed a distributed decision policy for influencing the aggregate number of EVs. Xia et al. [14] proposed an aggregator-based interactive charging management system for EV charging. Basically, the system is hierarchical because the EV management center coordinates with the distribution automation system to get the charging dispatching commands and send them to aggregators. Khan et al. [15] also investigated a hierarchical method, in which a bi-level online interaction procedure from the distribution system operator to the aggregators was presented and a water-filling algorithm in a two-step EV power allocation employed by the aggregators employ was introduced.

Each of these studies [9-15] is regarded as an important step forward for dispatching the CS with the decentralized or hierarchical method. The time-of-use (TOU) electricity price rewards customers who shift their usage away from on-peak hours and promotes more efficient use of energy resources. Moreover, the classical method could be an effective solution to such an emerging problem. The 
Lagrange relaxation method (LRM) is effective for performing decentralized optimization that has the features of simplicity, flexibility, and reliability [16]. Because the computational dimension of the original problem can be reduced, the LRM has been widely and successfully applied in the economic dispatch of the power generation system [17-20]. In addition, with the application of the LRM, it is unnecessary to have information interaction between contiguous sub-modules. In other words, if an error appears between the central controller and a local processor, then the optimization of other sub-modules is not disturbed. However, no report in the literature has provided a detailed illustration and its corresponding discussion of applying the LRM to large-scale EVs charging dispatch.

According to the aforementioned considerations, this study aims to present in detail a novel decentralized optimization of dispatching the CS for EVs with the application of LRM and TOU strategy. The main contributions of this paper can be summarized as follows:

(1) A decentralized control scheme is proposed in which the central controller (CC) is responsible for exchanging data with the local controllers (LCs) and regulating the overall framework. The optimization problem is divided into a set of sub-problems that are solved in parallel by the LCs. This will benefit the CC to lower processing burden and higher computation rate.

(2) Application of LRM in the decentralized optimization is presented in detail. With LRM, the conventional centralized optimization model into a set of $N_{E V}$ sub-problems, where $N_{E V}$ denotes the number of EVs. Each sub-problem and its constraints are convex, and their convexity ensures the optimization convergence.

This study is organized into five sections. Following the introduction, a centralized optimization model for dispatching the CS is presented to lay a foundation for establishing the decentralized optimization model. Section 3 gives the detailed application of the LRM to the decentralized optimization. The decentralized control and optimization mechanism is also introduced in Section 3. A comparison and discussion based on a series of numerical simulations are presented in Section 4 . Finally, conclusions are drawn in Section 5.

\section{Model for Centralized Optimization}

\subsection{Mathematical Formulation of EV Charging Load}

Based on a national household travel survey undertaken in 2009 by the Federal Highway Administration of the Department of Transportation of the United States [21], the probability density functions for the time being connected to and disconnected from the grid and the daily driving distance of the EV can be determined [22,23]. To obtain the period of each EV to be charged, the data regarding the departure and arrival time can be randomly generated by using the Monte Carlo method with the given probability density function of the daily departure time and driving distance of an EV. The distribution of the time as an EV to be connected to and disconnected from the grid can be depicted in Figure 1 and mathematically expressed as Equations (1) and (2), respectively [22,23].

$$
f_{s}\left(t_{c}\right)=\left\{\begin{array}{cc}
\frac{1}{\sqrt{2 \pi} \sigma_{s}} \exp \left(-\frac{\left(t_{c}+24-\mu_{s}\right)^{2}}{2 \sigma_{s}^{2}}\right) & 0<t_{c} \leq \mu_{s}-12 \\
\frac{1}{\sqrt{2 \pi} \sigma_{s}} \exp \left(-\frac{\left(t_{c}-\mu_{s}\right)^{2}}{2 \sigma_{s}^{2}}\right) & \mu_{s}-12<t_{c} \leq 24
\end{array},\right.
$$

where $t_{c}$ is the time as the EV to be connected to the grid; $\mu_{s}=17.1$; and $\sigma_{s}=3.3$.

$$
f_{e}\left(t_{l}\right)=\left\{\begin{array}{cc}
\frac{1}{\sqrt{2 \pi} \sigma_{e}} \exp \left(-\frac{\left(t_{l}-\mu_{e}\right)^{2}}{2 \sigma_{e}^{2}}\right) & 0<t_{l} \leq \mu_{e}+12 \\
\frac{1}{\sqrt{2 \pi} \sigma_{e}} \exp \left(-\frac{\left(t_{l}-24-\mu_{e}\right)^{2}}{2 \sigma_{e}^{2}}\right) & \mu_{e}+12<t_{l} \leq 24
\end{array},\right.
$$

where $t_{l}$ is the time as the EV to be disconnected from the grid; $\mu_{e}=8.92 ;$ and $\sigma_{e}=3.24$. 


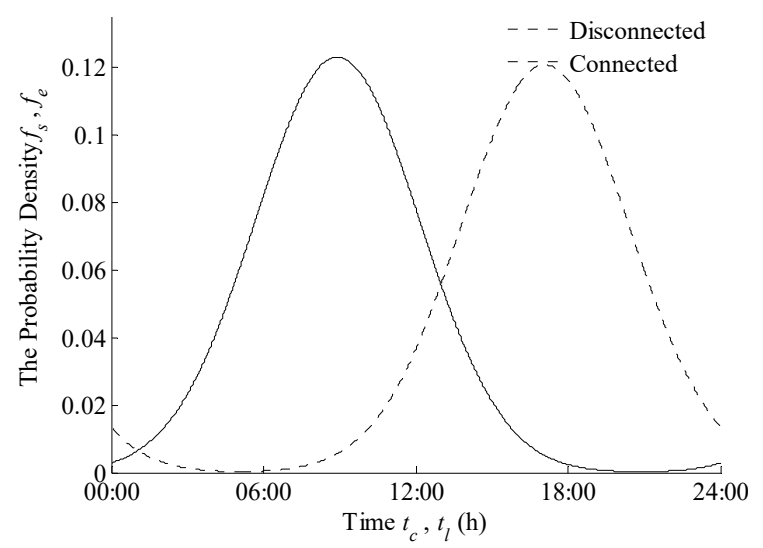

Figure 1. Distribution of the time of EVs to be connected to and disconnected from the grid.

The probability density function of the EV's daily driving distance $\left(R_{d}\right)$ is approximately regarded as a logarithmic normal distribution illustrated in Figure 2 and can be expressed as

$$
f_{m}\left(R_{d}\right)=\frac{1}{2 \pi \sigma_{m} R_{d}} \exp \left(-\frac{\left(\ln R_{d}-\mu_{m}\right)^{2}}{2 \sigma_{m}^{2}}\right)
$$

where $\mu_{m}=3.31$, and $\sigma_{m}=0.87$.

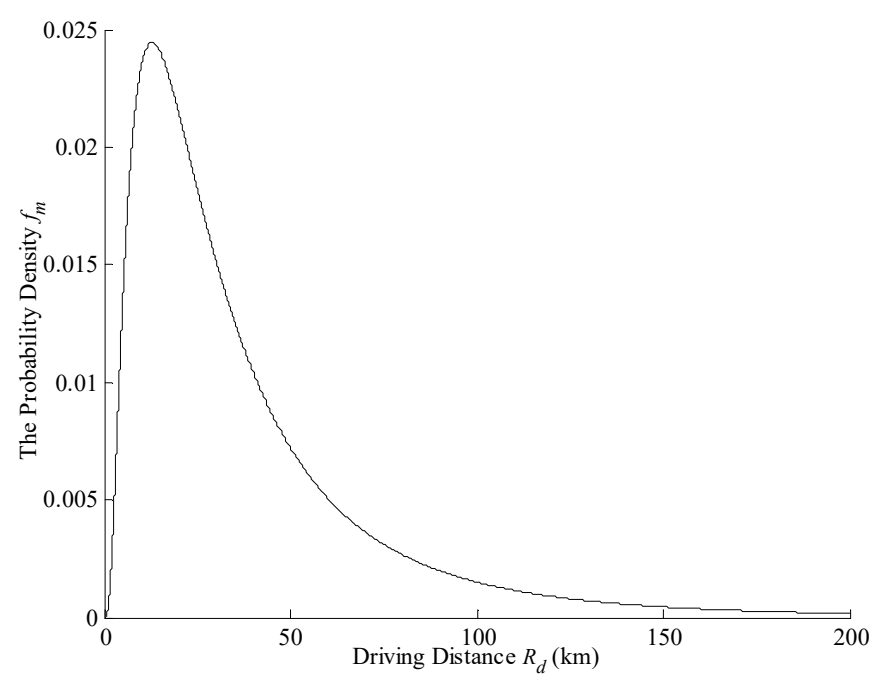

Figure 2. Lognormal distribution of the daily driving distance of the EV.

Suppose that once an EV is connected to the grid, it submits to the dispatch arrangement of the CSs. According to the expected state of charge (SOC) as the EV to be disconnected from the grid $\left(S O C_{e}\right)$ and the journey ahead, the initial SOC as the EV to be connected to the grid $\left(S O C_{S}\right)$ can be calculated as

$$
S O C_{S}=S O C_{e}-\frac{R_{d} E_{d 100}}{100 C_{c}}
$$

where $C_{c}$ denotes the power capacity of an EV battery, and $E_{d 100}$ represents the required electric energy for the distance of $100 \mathrm{~km}$.

If the charging demand of the $i^{\text {th }} \mathrm{EV}$ owner can be satisfied, then the values of $S O C_{s}$ and $S O C_{e}$, which are percentage points that must meet the constraint expressed by Equation (5). If the constraint is not qualified, then it is recommended that the EV owner modify $t_{l, i}$. 


$$
\frac{S O C_{e, i}-S O C_{s, i}}{P_{e}} \leq t_{l, i}-t_{c, i}
$$

where $P_{e}$ is the rated charging power in $\mathrm{kW}$; and $t_{c, i}$ and $t_{l, i}$ represent the time of the $i^{\text {th }} \mathrm{EV}$ are to be connected to and disconnected from the grid, respectively.

\subsection{Centralized Control Scheme and Optimization Model}

\subsubsection{Control Structure}

The centralized control structure of the CS for EVs is depicted in Figure 3. First, the CC collects the EVs' data, including $t_{c, i}, t_{l, i}, S O C_{e, i}$, and $S O C_{s, i}$, where the subscript " $i$ " represents the $i^{\text {th }} \mathrm{EV}$. Next, the $\mathrm{CC}$ sends the battery charging order to each EV, namely, the charging state during the battery charging period, after implementing the centralized optimization strategy. The mechanism is relatively simple and easy to realize; however, one of the significant problems is the computational complexity of the increasing number of EVs.

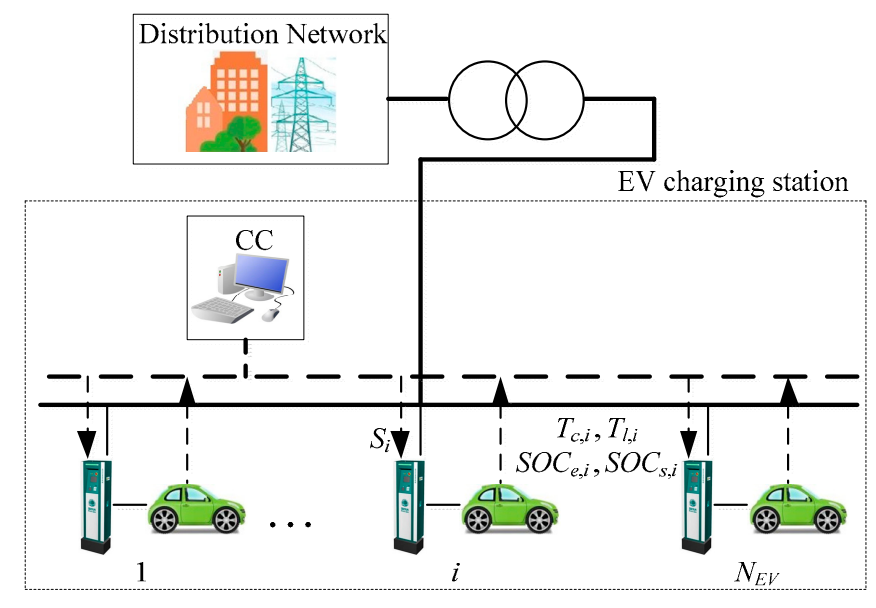

Figure 3. Centralized control architecture.

\subsubsection{Objective Function}

It is assumed that each day is divided equally into $N_{T}$ periods and that each period is set as $\Delta T$. The maximization of the charging profits in the CS is selected as the optimization objective function, which can be expressed as

$$
\max F=\sum_{i=1}^{N_{E V}} \sum_{j=1}^{N_{T}} P_{e} S_{i}^{j} \Delta T\left(g_{j}-p_{j}\right)
$$

where $F$ represents the daily earning; $N_{E V}$ is the number of $E V s ; g_{j}$ and $p_{j}$ denote the electricity prices that the CS sells to the EV owner and purchases from the grid in the $j^{\text {th }}$ period, respectively; and $S_{i}{ }^{j}$ denotes the charging state of the $i^{\text {th }} \mathrm{EV}$.

\subsubsection{Constraints}

(1). Charging demand of $i^{\text {th }} \mathrm{EV}$

$$
S O C_{e, j} C_{c} \leq S O C_{s, j} C_{c}+\sum_{j=1}^{N_{T}} P_{e} \eta S_{i}^{j} \Delta T \leq C_{c}
$$

where $\eta$ is the efficiency of the battery charging. The restriction in Equation (7) indicates that the energy at the end of the charging must be greater than that of the EV owner's expectation, and the SOC of the EV is less than its rated value. 


\section{(2). Acceptable charging periods}

It is reasonable to assume that an EV is scheduled to charge at the end of the period as the EV to be connected to the grid. Similarly, the battery-charging action is stopped at the end of the period before the EV is disconnected from the grid. The formulation of these two assumptions can be described as

$$
J_{c, i}=\left|\frac{t_{c, i}}{\Delta T}\right|, J_{l, i}=\left|\frac{t_{l, i}}{\Delta T}\right|
$$

where I I indicates the operator of rounding a number down, and $J_{c, i}$ and $J_{l, i}$ are the periods, where the $i^{\text {th }} \mathrm{EV}$ is connected to and disconnected from the grid, respectively. As illustrated in Figure 4, the acceptable charging period must be between $J_{c, i}$ and $J_{l, i}$. In other words, $S_{i}{ }^{j}$ in (6) can be formulated as

$$
S_{i}^{j}= \begin{cases}1 & j=J_{c, i}+1, \ldots, J_{l, i}-1 \\ 0 & j=1, \ldots, J_{c, i} J_{l, i}, \ldots, N_{T}\end{cases}
$$

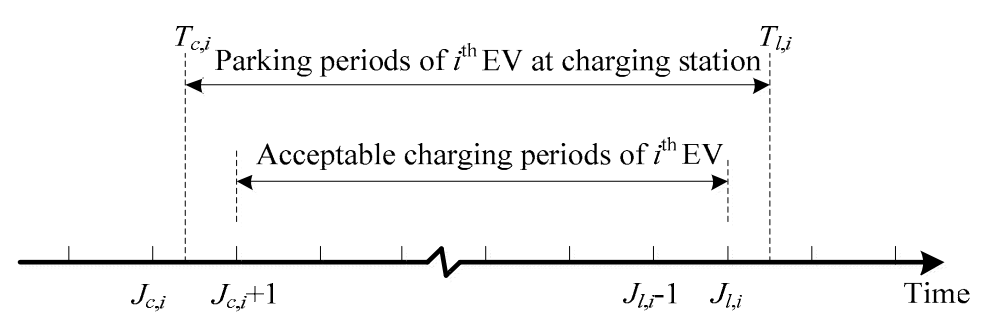

Figure 4. Diagram of acceptable charging periods.

(3). Added charging load

At any moment, the sum of the added charging load with the base load must be less than the following allowable maximum load:

$$
L_{0, j}+\sum_{i=1}^{N_{E V}} P_{e} S_{i}^{j}<P_{M}
$$

where $L_{0, j}$ is the base load of the distribution network in the $j^{\text {th }}$ period, and $P_{M}$ is the maximum load allowable in the system.

\section{Formulation of Decentralized Optimization}

\subsection{Decentralized Control and Optimization Architecture}

Different from the centralized scheme illustrated in Figure 3, the decentralized control architecture has local controllers (LCs). The structure diagram of the decentralized control can be depicted in Figure 5. The CC represents the superior scheduling controller of an integrated CS or a micro-grid that is responsible for exchanging data with the LCs to further handle the calculation results of the LCs synthetically and regulate the overall control framework. The CC in the decentralized scheme is distinct from that in the centralized architecture because of the reduced computational dimension. This process will result in lower processing burden and higher computation rate. In contrast, the LCs are often situated in the charging points and receive the load information from EV users directly. Moreover, the LCs can implement parallel computation based on the decentralized algorithm independently, determine the dispatch schemes for partial EVs, and deliver the corresponding dispatch schemes to the CC, possibly alleviating the calculation burden and the communication pressure broadly. 


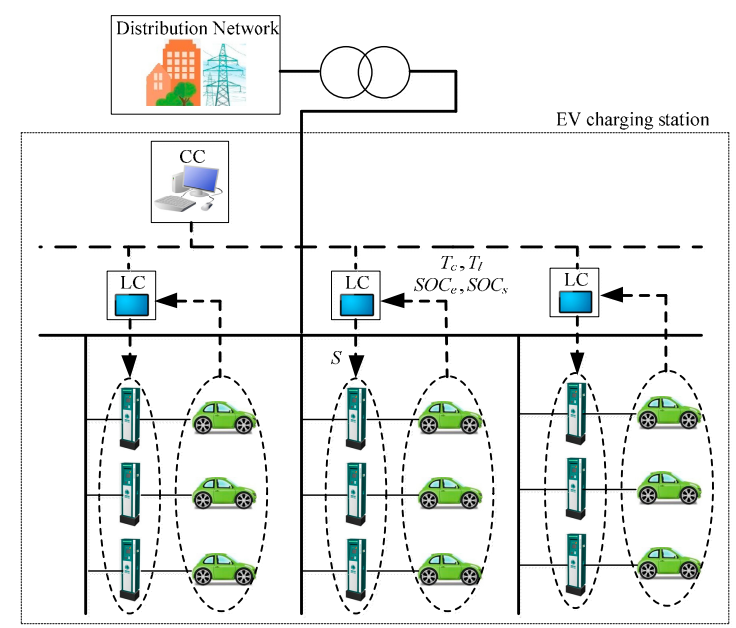

Figure 5. Decentralized control architecture.

\subsection{Application of LRM to Decentralized Optimization}

The optimization model described in Section 2 is suitable for the centralized optimization algorithm, which is difficult to apply to the decentralized model directly. One of the feasible approaches is to convert the centralized optimization model into a set of sub-problems to establish an appropriate decentralized model that should be in the form of mathematical expression of the decentralized optimization.

Equation (6) can be rewritten in the following form with the minimization operator:

$$
\min F=\sum_{i=1}^{N_{e v}} \sum_{j=1}^{N_{T}} P_{e} S_{i}^{j} \Delta T\left(p_{j}-g_{j}\right)
$$

The minimization problem mathematically expressed by Equation (11) serves as the original problem. With the application of the Lagrange relaxation method (LRM), this problem can be solved by identifying a set of solutions to the sub-problems via the computation method illustrated in Figure 6, as described in detail below.

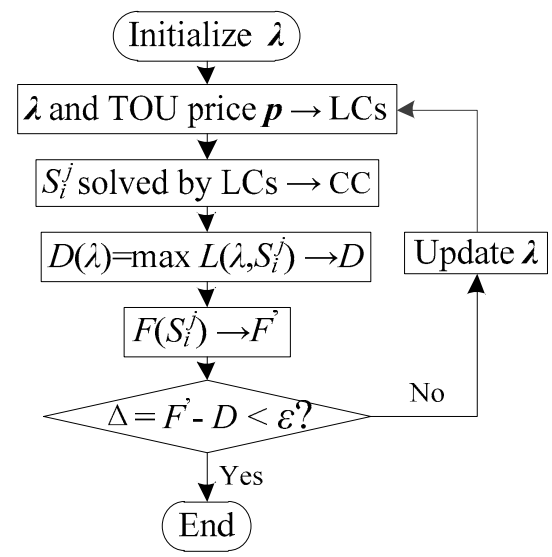

Figure 6. Flowchart of decentralized optimization of dispatching CS.

\subsubsection{Identification of Sub-Problems}

According to the Lagrange relaxation principle, Equation (10) is treated as the penalty term to be added into the original optimization objective function Equation (11). Moreover, the following formula, which is the corresponding Lagrange relaxation problem of the original problem, can be obtained: 


$$
\begin{gathered}
L\left(S_{i}^{j}, \lambda\right)=\sum_{i=1}^{N_{e v}} \sum_{j=1}^{N_{T}} P_{e} S_{i}^{j} \Delta T\left(p_{j}-g_{j}\right)+\sum_{j=1}^{N_{T}} \lambda_{i}\left(L_{0, j}+\sum_{i=1}^{N_{E V}} P_{e} S_{i}^{j}-P_{M}\right) \\
=P_{e} \sum_{i=1}^{N_{e v}} \sum_{j=1}^{N_{T}}\left[\Delta T\left(p_{j}-g_{j}\right)+\lambda_{i}\right] S_{i}^{j}+\sum_{j=1}^{N_{T}} \lambda_{i}\left(L_{0, j}-P_{M}\right)
\end{gathered}
$$

where $\lambda_{j}$ are the Lagrange multipliers, which affect the convergence of the decentralized optimization algorithm, as will be discussed in detail later.

The above Lagrange relaxation problem can be equivalently decomposed into $N_{E V}$ sub-problems, i.e., each of which corresponds to each EV.

$$
\min L\left(S_{i}^{j}, \lambda_{j}\right)=P_{e} \sum_{j=J_{c, j}+1}^{J_{L, i}-1}\left(\Delta T\left(p_{j}-g_{j}\right)+\lambda_{j}\right) S_{i}^{j}+\sum_{j=1}^{N_{T}} \lambda_{j}\left(L_{0, j}-P_{M}\right) / N_{E V}
$$

Apparently, Equation (13) is subjected to Equations (7) and (9), as can be integrated in the following expression:

$$
\operatorname{SOC}_{e, i} C_{c} \leq S O C_{s, i} C_{c}+\sum_{j=J_{c, i}+1}^{J_{l, i}-1} P_{e} \eta S_{i}^{j} \Delta T \leq C_{c}
$$

The above $N_{E V}$ sub-problems are easy to solve for obtaining the charging state variable $S_{i}^{j}$ for each EV.

\subsubsection{Solution to Dual Problem}

The dual problem of the original problem can be obtained when the Lagrange multipliers in the relaxation function are regarded as variables. The corresponding expression can be formulated as

$$
D(\lambda)=\max _{\lambda \geq 0} \min _{S_{i}^{j}} L\left(S_{i}^{j}, \lambda\right)
$$

\subsubsection{Computation of Upper and Lower Bounds for Original Problem}

Once the solution to the $i^{\text {th }}$ sub-problem, $\left.S_{i}{ }^{j}\right|_{i=1,2, \ldots, N E V, j=1,2, \ldots, N T}$, is identified, the objective function values of the original and its dual problems are calculated with the substitution of each $S_{i}{ }^{j}$ into Equations (11) and (15), which are the upper and lower bounds of the optimal solution to the original problem, $F^{\prime}$ and $D$, respectively.

\subsubsection{Evaluation of Dual Gap}

When the difference between the upper and lower bounds, $\Delta=F^{\prime}-D$, is no more than the defined accuracy $(\varepsilon), D$ can be approximately considered as the optimal solution of the original problem. Otherwise, one conducts the next step and updates the Lagrange multipliers by the sub-gradient algorithm.

\subsubsection{Updating of $\lambda$}

The Lagrange multipliers $\lambda$ can be updated according to Equation (16), where $k$ denotes the current iteration; $\gamma$ is the iteration step, whose expression is described by Equation $(17) ; \varphi(k)=\left[\varphi_{1}(k), \cdots\right.$, $\left.\varphi_{N T}(k)\right]^{\mathrm{T}}$ represents the sub-gradient; $\|\boldsymbol{\varphi}\|^{1}$ is 1-norm of $\boldsymbol{\varphi}$; each component $\varphi_{j}(k)$ in $\boldsymbol{\varphi}$ can be computed according to Equation (18).

$$
\begin{gathered}
\lambda(k+1)=\lambda(k)+\gamma(k) \frac{\varphi(k)}{\|\varphi(k)\|^{1}} \\
\gamma(k)=\frac{1}{a+b k}
\end{gathered}
$$




$$
\varphi_{j}(k)=L_{0, j}+\sum_{i=1}^{N_{E V}} P_{e} S_{i}^{j}-P_{M}, j=1, \ldots, N_{T}
$$

Parameters ( $a$ and $b$ ) in Equation (17) are constant and should satisfy the condition of $b<a$. Notably, these parameters must be appropriately selected for good convergence and can be determined by the trial-and-error process during the simulation. The procedure can be implemented in a practical dispatching system.

Apparently, with the application of LRM, the original centralized optimization problem is divided into $N_{E V}$ sub-problems formulated by Equation (13), subject to Equation (14). Because both the optimization objective-function and its constraint are convex, the optimization algorithm is able to converge to the optimal solution according to the theory of the convex optimization. Moreover, it is obvious that the performance of the decentralized optimization algorithm is related to the Lagrange multiplier, $\lambda$. The corresponding discussion will be given in Section 4.

\section{Simulations and Results}

To demonstrate the feasibility of the proposed decentralized model and the effectiveness of the decentralized optimization algorithm based on the LRM as well as to obtain a deep insight into the effect of applying the TOU price on the profit of the CS and peaking clipping/valley filling of the load curve, various case studies along with analysis and discussion to be conducted based on the variable values and the TOU price [24] are presented in Tables 1 and 2, respectively. The centralized optimization model is solved by using the Matlab software with the introduction of CPLEX [25]. Moreover, the uncontrolled charging [26] refers to the instant charging, i.e., EVs are successively charged immediately when they arrive at the CS, and the battery charging continues until their expected charging energy is achieved. It is assumed that the number of EVs arriving at the charging station during the scheduling is 150.

Case 1 (Proposed): Decentralized optimization and TOU price strategy.

Case 2: Decentralized optimization and fixed price strategy.

Case 3: Centralized optimization and TOU price strategy.

Case 4: Centralized optimization and fixed price strategy.

Case 5: Uncontrolled charging and TOU price strategy.

Case 6: Uncontrolled charging and fixed price strategy.

Table 1. Parameter values.

\begin{tabular}{cccc}
\hline Variable & Value & Variable & Value \\
\hline$N_{T}$ & 96 & $P_{e}$ & $7 \mathrm{~kW}$ \\
$\Delta T$ & $15 \mathrm{~min}$ & $\eta$ & 0.95 \\
$P_{M}$ & $5087 \mathrm{~kW}$ & $E d_{100}$ & $15 \mathrm{~kW} \cdot \mathrm{h}$ \\
$C_{c}$ & $32 \mathrm{~kW} \cdot \mathrm{h}$ & $S O C_{e}$ & $95 \%$ \\
\hline
\end{tabular}

Table 2. TOU price set by grid and CS.

\begin{tabular}{ccc}
\hline Time Interval & $p_{j} /$ CNY (kWh) $)^{-\mathbf{1}}$ & $g_{j} /$ CNY (kWh) $)^{-1}$ \\
\hline 00:00-08:00 & 0.365 & 0.4 \\
08:00-12:00 & 0.869 & 2.0 \\
12:00-14:30 & 0.687 & 1.2 \\
14:30-17:00 & 0.687 & 2.0 \\
17:00-21:00 & 0.869 & 2.0 \\
21:00-24:00 & 0.687 & 1.2 \\
\hline
\end{tabular}

The corresponding charging load curves are illustrated in Figure 7, in which the basic and total loads refer to the local electric power load without and added with EV charging load, respectively. Moreover, the profits of the CS corresponding to each case are summarized in Table 3. For simplicity, the fixed price given by the CS is set to be 1 CNY. 


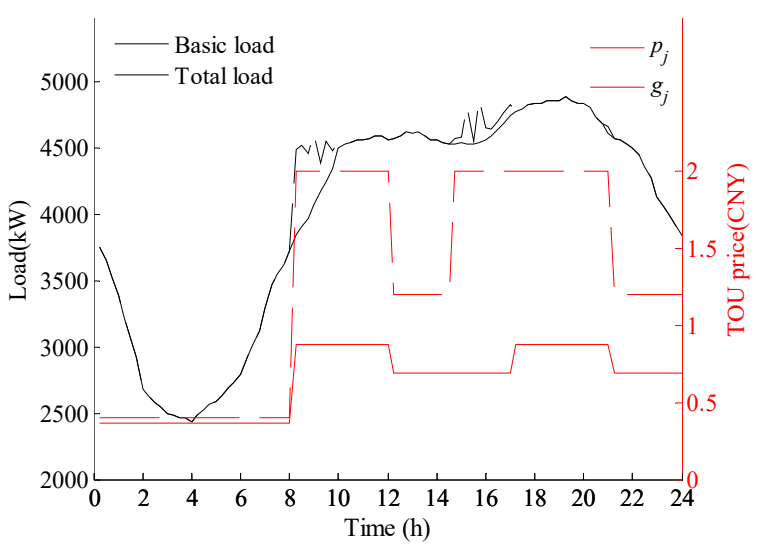

(a)

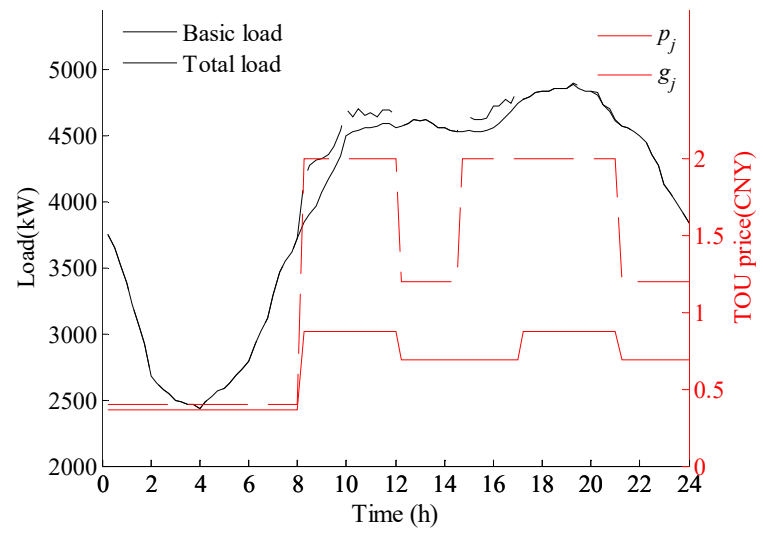

(c)

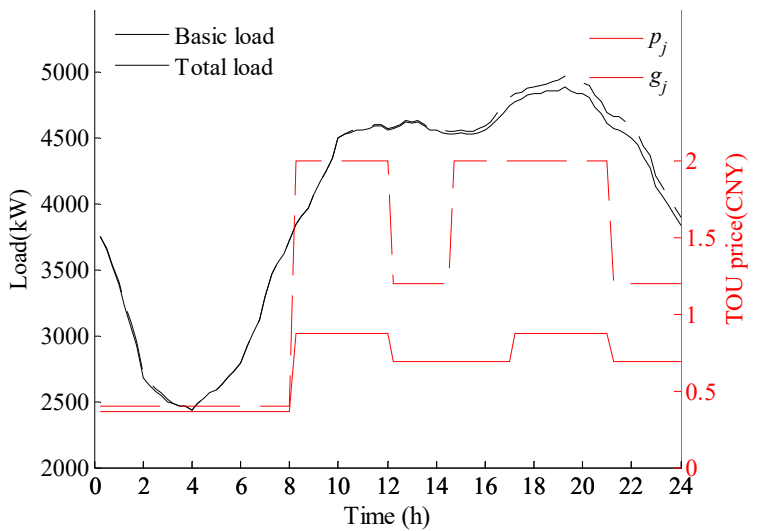

(e)

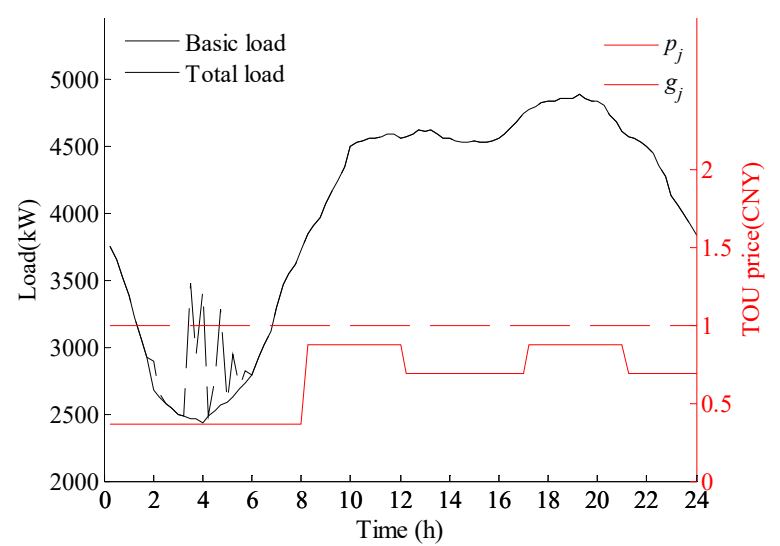

(b)

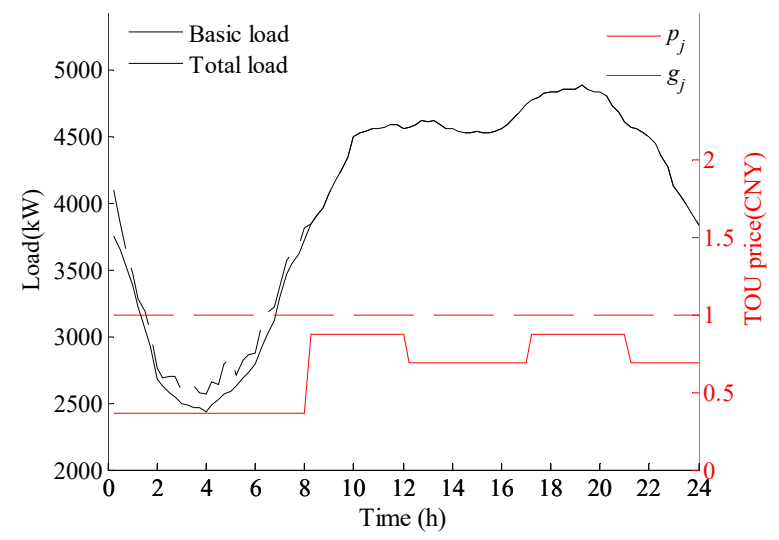

(d)

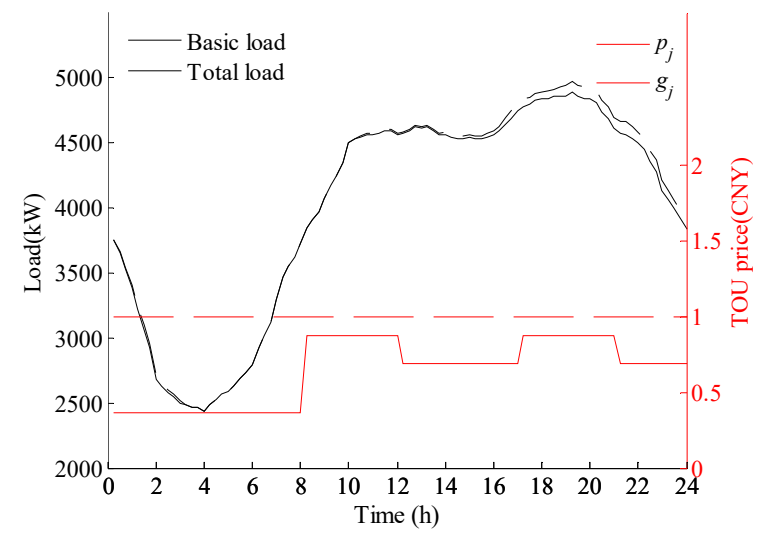

(f)

Figure 7. Charging load curve corresponding to (a) Case 1; (b) Case 2; (c) to Case 3; (d) Case 4; (e) Case 5 ; (f) Case 6 .

Table 3. Profit of charging station corresponding to each case.

\begin{tabular}{cccc}
\hline Case & $\boldsymbol{F ( C N Y )}$ & Case & $\boldsymbol{F ( C N Y )}$ \\
\hline Case 1 & 1348.375 & Case 4 & 654.526 \\
Case 2 & 644.526 & Case 5 & 796.749 \\
Case 3 & 1353.375 & Case 6 & 240.599 \\
\hline
\end{tabular}

Firstly, the inspection of the result illustrated in Figure 5a shows that the proposed method with the decentralized optimization and the TOU price strategy reasonably generates two charging 
periods, namely, 8:00 to 10:00 and 14:00 to 18:00. Such solution is identified because of the optimization objective, that is, maximization of the CS profit, and the larger difference between the two TOU price schemes during these two periods, as investigated from Table 2. This approach is quite different from the uncontrolled charging (Case 5) that increases the original peak load because of the disorderly EV charging load, as illustrated in Figure 5e. In contrast, compared with that of the centralized optimization (Case 3) demonstrated in Figure 5c, the optimized load curve has newly generated peak loads in the charging periods. Hence, regarding the compensation of the load fluctuation, the effect of the decentralized method is worse than the centralized method. Note that, because of the constraint expressed by Equation (10), the newly generated peak loads are in the allowable range.

Secondly, simulated results demonstrated that the identified optimization solution is significantly related to the pricing strategy and that the TOU strategy is helpful for realizing the CS's profit. After applying the fixed charging electricity price, both the decentralized (Case 2) and centralized (Case 4) methods schedule the charging in the period of 0:00 to 8:00, which is also the load-valley period. The difference lies in the charging distribution. The distribution is largely concentrated in 3:00-5:00 for the decentralized method (Case 2) and covers the whole period for the centralized method (Case 4). As in the added charging load curve in Case 1, the effect of valley-filling resulted from the decentralized method is worse than the centralized method in the case of applying the fixed price strategy. For the uncontrolled charging method, the EV charging behavior is not affected by the pricing strategy, as shown in Figure 5e,f. However, overall, as indicated in Figure 5, because of the difference between the price formulated by the CS and the TOU tariff in the grid, the CS's profits corresponding to the TOU strategy, namely, Case 1, Case 3 and Case 5, are more than those of the fixed price strategy, namely, Case 2, Case 4, and Case 6. Clearly, with the TOU strategy, the profits of the decentralized and centralized optimization are approximately $1350 \mathrm{CNY}$. With the fixed strategy, the profit is approximately $650 \mathrm{CNY}$, which is $48.1 \%$ of the TOU strategy.

Additionally, another three cases that the rated capacities of EV are $24 \mathrm{~kW} \cdot \mathrm{h}, 32 \mathrm{~kW} \cdot \mathrm{h}$ and $40 \mathrm{~kW} \cdot \mathrm{h}$ have been considered. The corresponding charging loads are plotted in Figure 8 as shown below. Apparently, the charging load curves are similar with that of $C c=32 \mathrm{~kW} \cdot \mathrm{h}$ demonstrated in Figure $7 \mathrm{a}$. They are different from each other in that the charging load in the charging periods is increasing with the rated capacity.

Thirdly, numerical simulations with various amounts of EVs show the computation efficiency and stability of the decentralized optimization. The computational efficiency comparison of the centralized control and the decentralized control for various amounts of EVs is depicted in Figure 9. The figure demonstrates that the computation time of the decentralized optimization (Case 3) almost remains constant when the number of EVs increases. Regarding the centralized optimization (Case 4), the computation time dramatically increases as the number of EVs increases. Hence, it can be concluded that the proposed decentralized optimization has unique superiority regarding computation requirement and can be used for the online charging dispatch.

Fourthly, as mentioned earlier, the convergence performance of the proposed decentralized optimization algorithm is related to the Lagrange multipliers $\lambda_{0}$. To analyze the effect of $\lambda_{0}$ on the convergent property, this study takes Case 1 as an example, and all the initial multipliers $\left[\lambda_{0}\right]_{1 \times 96}$ are set as the same value for simplification on the analysis. The time spent for solving the decentralized model and the numbers of iteration at different values of $\lambda_{0}$ are determined, as illustrated in Figure 10. The simulated result reveals that less computation time is acquired with the smaller values of $\left[\lambda_{0}\right]_{1 \times 96}$ when the initial values of Lagrange multipliers varies within a certain range of $\left[5 \times 10^{-3}, 11 \times 10^{-3}\right]$. Therefore, the result implies that appropriate values of initial Lagrange multipliers $\left[\lambda_{0}\right]_{1 \times 96}$ directly improve the convergence rate of the proposed decentralized algorithm. 


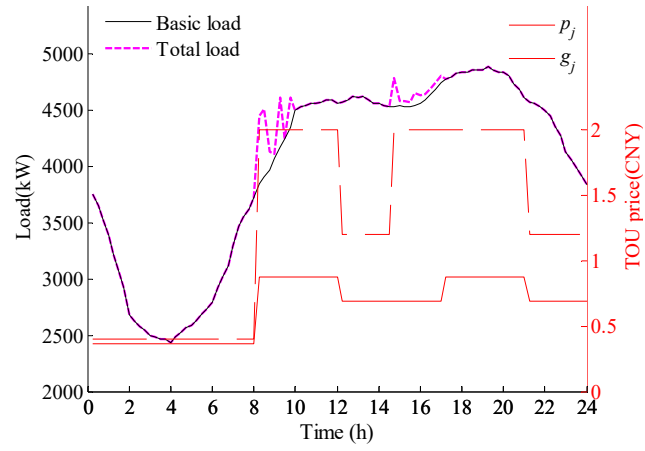

(a)

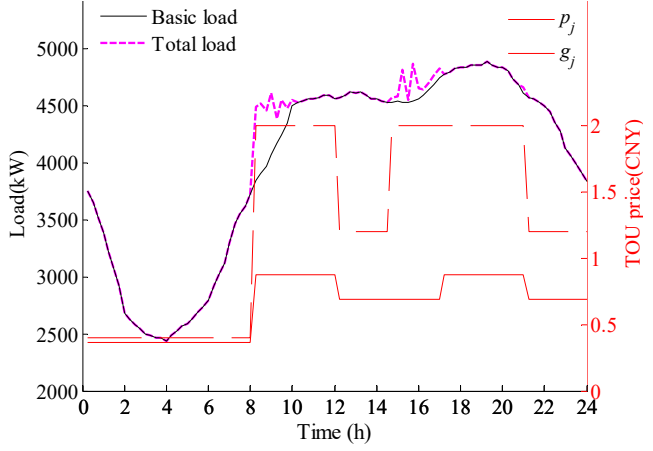

(b)

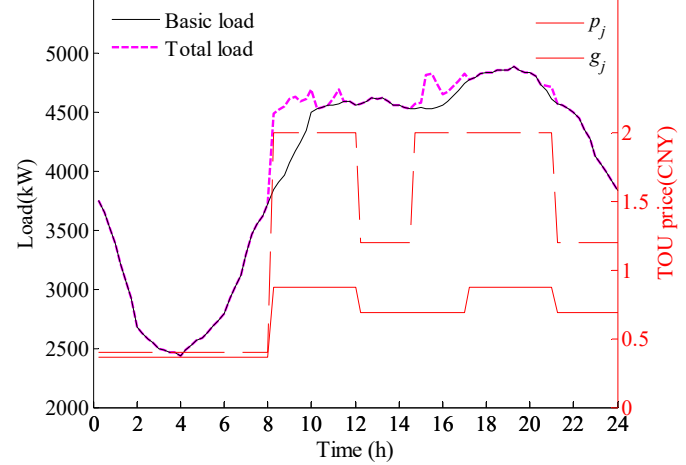

(c)

Figure 8. Charging load curve corresponding to $C c=(\mathbf{a}) 24 ;(\mathbf{b}) 32 ;$ (c) 40.

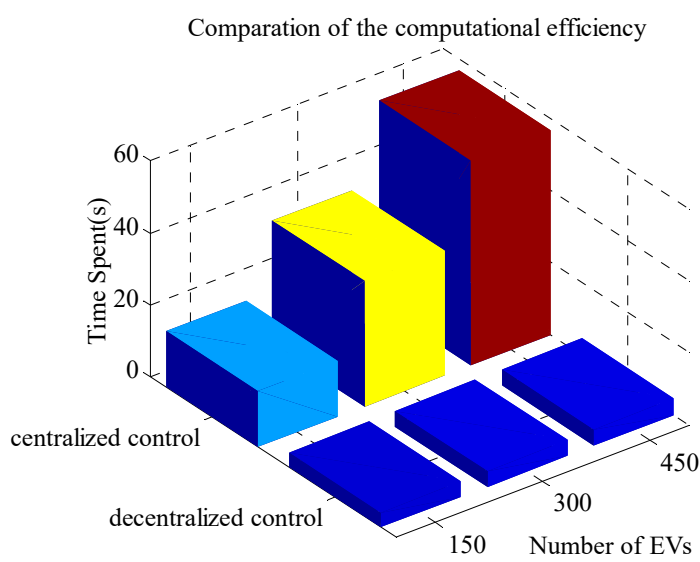

Figure 9. Comparison of computational efficiency.

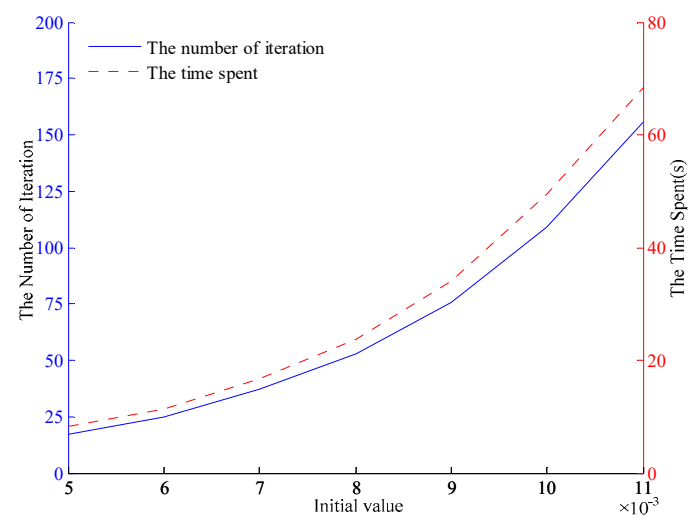

Figure 10. Relationship between the initial value $\lambda_{0}$ and the convergence rate. 


\section{Conclusions}

This study successfully provided a decentralized solution for the optimal dispatch problem of the CS for EVs by utilizing the LRM and the TOU price strategy. This proposed approach converts the conventional centralized optimization problem into a set of sub-problems. The proposed method can effectively utilize sparse communication infrastructures with limited message passing among adjacent communication units and result in unique superiority regarding computation time. The effectiveness of the proposed method was demonstrated by numerical simulations with various amount of EVs and different price strategies and compared with the results performed by the centralized optimization and the uncontrolled charging method. The simulated results demonstrated that the proposed approach has very satisfactory computation efficiency performance. In comparison with the charging load curve of the centralized optimization, the proposed decentralized optimization does not perform better. In spite of the excellent convergence, the performance of the proposed decentralized optimization is likely to be related to the initial Lagrange multipliers. In future research, this method will be continuously optimized to realize a better effect of the peak-cutting and valley-filling of the power use and extend it to the online dispatching of the CS.

Author Contributions: The entire research work has been carried out by Y.F. and X.W. under the guidance of S.C. The individual contributions of the authors are specified as follows: Conceptualization, S.C.; Methodology, S.C. and X.W.; Software, Y.F. and X.W.; Validation, Y.F. and X.W.; Writing-Original Draft Preparation, Y.F. and X.W.; Writing-Review \& Editing, S.C. and Y.F.; Funding Acquisition, S.C.

Funding: This research was funded by the National Natural Science Foundation of China under grant no. 51607105.

Conflicts of Interest: The authors declare no conflict of interest.

\section{References}

1. Yuan, X.; Liu, X.; Zuo, J. The development of new energy vehicles for a sustainable future: A review. Renew. Sustain. Energy Rev. 2015, 42, 298-305. [CrossRef]

2. Yan, X.; Crookes, R.J. Life cycle analysis of energy use and greenhouse gas emissions for road transportation fuels in China. Renew. Sustain. Energy Rev. 2009, 13, 2505-2514. [CrossRef]

3. Liu, H.; Zeng, P.; Guo, J.; Wu, H.; Ge, S. An optimization strategy of controlled electric vehicle charging considering demand side response and regional wind and photovoltaic. J. Mod. Power Syst. Clean Energy 2015, 3, 232-239. [CrossRef]

4. Cortés, A.; Martínez, S. A hierarchical algorithm for optimal plug-in electric vehicle charging with usage constraints. Automatica 2016, 68, 119-131. [CrossRef]

5. Kang, Q.; Wang, J.B.; Zhou, M.C.; Ammari, A.C. Centralized charging strategy and scheduling algorithm for electric vehicles under a battery swapping scenario. IEEE Trans. Intell. Transp. Syst. 2016, 17, 659-669. [CrossRef]

6. Ou, C.H.; Liang, H.; Zhuang, W. Investigating wireless charging and mobility of electric vehicles on electricity market. IEEE Trans. Ind. Electron. 2015, 62, 3123-3133. [CrossRef]

7. Korkas, C.D.; Baldi, S.; Yuan, S.; Kosmatopoulos, E.B. An adaptive learning-based approach for nearly optimal dynamic charging of electric vehicle fleets. IEEE Trans. Intell. Transp. Syst. 2017, 19, 2066-2075. [CrossRef]

8. Luo, Y.; Zhu, T.; Wan, S.; Zhang, S.; Li, K. Optimal charging scheduling for large-scale EV (electric vehicle) deployment based on the interaction of the smart-grid and intelligent-transport systems. Energy 2016, 97, 359-368. [CrossRef]

9. Karfopoulos, E.L.; Hatziargyriou, N.D. A multi-agent system for controlled charging of a large population of electric vehicles. IEEE Trans. Power Syst. 2013, 28, 1196-1204. [CrossRef]

10. Xydas, E.; Marmaras, C.; Cipcigan, L.M. A multi-agent based scheduling algorithm for adaptive electric vehicles charging. Appl. Energy 2016, 177, 354-365. [CrossRef]

11. García-Triviño, P.; Torreglosa, J.P.; Fernández-Ramírez, L.M.; Jurado, F. Control and operation of power sources in a medium-voltage direct-current microgrid for an electric vehicle fast charging station with a photovoltaic and a battery energy storage system. Energy 2016, 115, 38-48. [CrossRef] 
12. Wang, D.; Guan, X.; Wu, J.; Li, P.; Zan, P.; Xu, H. Integrated energy exchange scheduling for multimicrogrid system with electric vehicles. IEEE Trans. Smart Grid 2016, 7, 1762-1774. [CrossRef]

13. Gusrialdi, A.; Qu, Z.; Simaan, M.A. Distributed scheduling and cooperative control for charging of electric vehicles at highway service stations. IEEE Trans. Intell. Transp. Syst. 2017, 18, 2713-2727. [CrossRef]

14. Xia, M.; Lai, Q.; Zhong, Y.; Li, C.; Chiang, H.-D. Aggregator-Based Interactive Charging Management System for Electric Vehicle Charging. Energy 2016, 9, 159. [CrossRef]

15. Khan, S.U.; Mehmood, K.K.; Haider, Z.M.; Rafique, M.K.; Kim, C.-H. A Bi-Level EV Aggregator Coordination Scheme for Load Variance Minimization with Renewable Energy Penetration Adaptability. Energy 2018, 11, 2809. [CrossRef]

16. Merlin, A.; Sandrin, P. A new method for unit commitment at electricité de France. IEEE Trans. Power Appar. Syst. 1983, 102, 1218-1225. [CrossRef]

17. Lai, X.; Xie, L.; Xia, Q.; Zhong, H.; Kang, C. Decentralized multi-area economic dispatch via dynamic multiplier-based Lagrangian relaxation. IEEE Trans. Power Syst. 2015, 30, 3225-3233. [CrossRef]

18. Lai, X.; Zhong, H.; Xia, Q.; Kang, C. Decentralized intraday generation scheduling for multiarea power systems via dynamic multiplier-based Lagrangian relaxation. IEEE Trans. Power Syst. 2017, 30, 3225-3233. [CrossRef]

19. Ding, T.; Bie, Z. Parallel augmented Lagrangian relaxation for multi- period economic dispatch using diagonal quadratic approximation method. IEEE Trans. Power Syst. 2017, 32, 1115-1126. [CrossRef]

20. Hua, B.; Baldick, R.; Wang, J. Representing operational flexibility in generation expansion planning through convex relaxation of unit commitment. IEEE Trans. Power Syst. 2017, 33, 2272-2281. [CrossRef]

21. US Department of Transportation, Federal Highway Administration. 2009 national household travel survey. Available online: http//nhts.ornl.gov/2009/pub/stt.pdf (accessed on 18 March 2018).

22. Tian, L.; Shi, S.; Jia, Z. A Statistical Model for Charging Power Demand of Electric Vehicles. Power Syst. Technol. 2010, 34, 126-130. [CrossRef]

23. Xu, S.; Feng, D.; Yan, Z.; Zhang, L.; Li, N.; Jing, L.; Wang, J. Ant-Based Swarm Algorithm for Charging Coordination of Electric Vehicles. Int. J. Distrib. Sens. Netw. 2013, 9, 268942. [CrossRef]

24. Sun, X.; Wang, W.; Su, S.; Jiang, J.C.; Xu, L.J.; He, X.H. Coordinated charging strategy for electric vehicles based on time-of-use price. Autom. Electr. Power Syst. 2013, 37, 191-195. [CrossRef]

25. Zhang, L.; Yan, Z.; Feng, D.H.; Xu, S.L.; Li, N.H.; Jing, L. Two-stage optimization model based coordinated charging for EV charging station. Power Syst. Technol. 2014, 38, 967-974. [CrossRef]

26. Pan, Z.N.; Zhang, X.S.; Yu, T.; Wan, D.Z. Hierarchical real-time optimized dispatching for large-scale clusters of electric vehicles. Autom. Electr. Power Syst. 2017, 41, 96-104. [CrossRef] 